\title{
Effects of Light-Scattering Coefficient of Adsorbent in Diffuse Reflectance Spectroscopy of Dye-Doped Microparticles
}

\author{
Noboru Kitamura ${ }^{\dagger}$, Shoji Ishizaka and Haeng-Boo KIM \\ Division of Chemistry, Graduate School of Science, Hokkaido University, Sapporo 060, Japan
}

\begin{abstract}
Diffuse reflectance spectroscopy on polymer particles and silica gels adsorbing tris(2,2'-bipyridine)ruthenium(II), Ru(II), was conducted to elucidate the shape and size effects of an adsorbent on the spectrum. The diffuse reflectance light intensity $\left(f\left(r_{\infty}\right)\right)$ was linearly correlated with the amount of the complex ([Ru(II)]) adsorbed on spherical polymer beads, amorphous-, or spherical-shaped silica gels as low as $[\mathrm{Ru}(\mathrm{II})] \sim 10^{-8} \mathrm{~mol} / \mathrm{cm}^{3}$. However, the slope of a $f\left(r_{\infty}\right)$ vs. $[\mathrm{Ru}(\mathrm{II})]$ plot was different between the adsorbents. The results were explained in terms of the different light scattering coefficient between the adsorbents, originating primarily from the different shape and size of the adsorbent.
\end{abstract}

Keywords Diffuse reflectance spectroscopy, Kubelka-Munk function, scattering coefficient, adsorbent morphology

Diffuse reflectance spectroscopy (DRS) is a unique method for analyzing various opaque materials such as colored glasses, organic and inorganic pigments, dyedoped polymer particles and so forth, and has been extensively studied since 1950 s. ${ }^{1,2}$ Besides DRS, photoacoustic or photothermal spectroscopy (PAS or PTS, respectively) can also be applied for analyzing solid materials, and has been known to be a sensitive method for determining the solute concentration in a material. In order to obtain spectral/electronic information about a solute, however, PAS or PTS is generally inconvenient, since the excitation wavelength dependence of a PAS or PTS signal (i.e., action spectrum) must be studied. ${ }^{3}$ On the other hand, since DRS can directly provide spectral/ electronic information about a solute in a material, the method plays important roles in various research fields. In analytical science, for example, DRS in the infrared (IR) or near-IR region has been applied to the detection of separated components in thin-layer chromatography ${ }^{4}$ as well as to the quantitation of active compounds in tablet-form drugs. ${ }^{3}$ The determination of functional groups on silica surfaces and kinetic analyses of the reactions between $\mathrm{NO}_{2}$ and synthetic sea salts have been also conducted by IR-DRS. ${ }^{6,7}$ Furthermore, timeresolved DRS in the UV-visible region has received current interests as a powerful technique for elucidating charge-transfer processes in molecular crystals ${ }^{8}$, semiconductor nanoparticles ${ }^{9}$, dye-doped zeolites ${ }^{10}$, and so forth. ${ }^{11}$

It has been well known that DRS is essentially based on observing a diffuse reflectance (DR) light intensity from a solid sample. Under the assumption that the sample layer is infinitely thick, the DR light intensity from a sample at a given wavelength can be written as in Eq. $(1)^{1,2}$,

$\dagger_{\text {To }}$ whom correspondence should be addressed.

$$
f\left(r_{\infty}\right)=\left(1-r_{\infty}\right)^{2} / 2 r_{\infty}=K / S
$$

where $r_{\infty}$ is the ratio of the reflectance light intensity of the sample to that of a reference sample. $K$ and $S$ represent absorption and light-scattering coefficients of the sample, respectively. The absorption coefficient is proportional, but not equal, to the relevant absorptivity determined by conventional transmission spectroscopy. According to the Kubelka-Munk theory ${ }^{1,2}, f\left(r_{\infty}\right)$ is proportional to the concentration of the absorbing species as long as the absorbances of the sample are not high. In practice, it has been reported that $f\left(r_{\infty}\right)$ is linearly correlated to the sample concentration up to $f\left(r_{\infty}\right)=3.5 .{ }^{12}$ Thus, DRS can be used for quantitative analyses of solid samples as mentioned above.

As a main drawback, on the other hand, DRS is based on light reflection by sample solids or particles, so that the reflected light intensity is dependent on $S$ of a sample, as expected from Eq. (1). This implies that an information obtained by DRS is governed by the size, shape, and surface morphology of a sample. Therefore, a direct comparison of the DR spectra between materials with different properties is generally difficult. Although magnesium oxide $(\mathrm{MgO})$ is commonly used for the determination of $S$ of a sample ${ }^{1,2}$, this method is not necessarily convenient, since freshly prepared $\mathrm{MgO}$ is required for precise measurements of $S$. The $S$ values of samples have thus been rarely discussed. For materials doped with a common dye, on the other hand, $K$ is essentially determined by the light absorption of the dye, while $S$ is governed by the properties of a material (i.e., adsorbent) itself. In dye/adsorbent systems, therefore, effects of the light-scattering coefficient of an adsorbent on the DR spectra can be studied in detail. We consider that such a study is very important for further applications of DRS to various opaque materials.

In this paper, we report on the UV-visible DRS of 


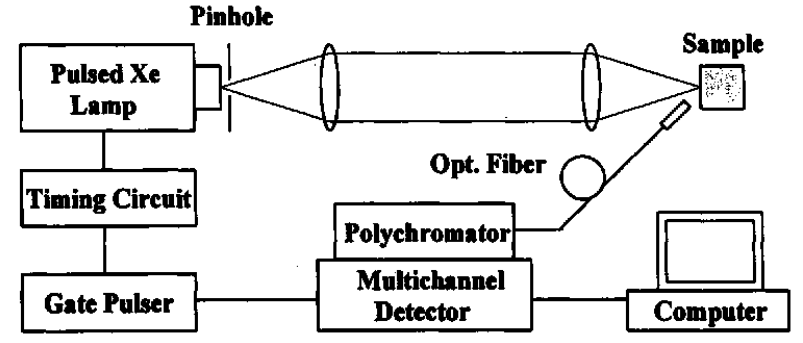

Fig. 1 Block diagram of the experimental setup for diffuse reflectance spectroscopy.

tris(2,2'-bipyridine)ruthenium(II) $(\mathrm{Ru}(\mathrm{II}))$ adsorbed on materials with different size, shape and morphology (i.e., spherical polymer beads, spherical-shaped silica gel, and amorphous-shaped silica gel) in order to elucidate the effects of the light-scattering coefficient of an adsorbent $(S)$ on the DR spectra. The effects of the distribution characteristics of $\mathrm{Ru}(\mathrm{II})$ inside spherical polymer beads on the spectra are also discussed.

\section{Experimental}

\section{Apparatus}

DRS was performed by the system illustrated in Fig. 1. For UV-visible DRS on Ru(II)/adsorbent systems, the samples strongly absorb incident light, so that the use of an intense light source is preferable. Therefore, we used a pulsed Xe flash lamp (Tokyo Instruments Inc., pulse width $=7 \mu \mathrm{s}, 150 \mathrm{~W}$ ) as a light source. The Xe lamp beam was irradiated perpendicularly to the sample cell, and diffuse reflectance light was introduced to an optical fiber. Although we monitored the diffuse reflectance light from the sample cell at various angles of the fiber, the most intense signals and the best signal/noise ratios were obtained when the optical fiber was set at $45^{\circ}$ to the sample cell, as shown in Fig. 1. Therefore, we performed all of the experiments under such optical alignments. Diffuse reflectance light was then analyzed by a polychromator (Jobin-Ybon, HR320)-photodiode array detector (Tokyo Instruments Inc., IRY-512) set. The timing between Xe flash lamp- and detectoroperations was controlled by a digital-delay generator (Stanford Research Systems Inc., DG535), and was set so as to monitor the most intense diffuse reflectance light from the sample. Analogous experiments can be performed using a commercially available spectrometer, equipped with a diffuse reflectance apparatus. However, we think that the present experimental setup, using an optical fiber and a photodiode array detector, is very convenient to conduct DRS. The $f\left(r_{\infty}\right)$ value was obtained by comparing the light intensity from a dyedoped sample $\left(r_{\mathrm{s}}\right)$ with that from a dye-free adsorbent $\left(r_{\mathrm{r}}\right)$ : $r_{\infty}=r_{s} / r_{\mathrm{r}}$. Conventional transmission spectroscopy for an aqueous $\mathrm{Ru}(\mathrm{II})$ solution $(10 \mathrm{~mm}$ optical path length cuvette) was conducted by using a Shimadzu UV-200S spectrophotometer.

\section{Chemicals and sample preparations}

Tris(2,2'-bipyridine)ruthenium(II) dichloride hexahydrate (Aldrich) was used as supplied. Three adsorbents with different sizes and shapes were used, as the relevant optical micrographs were shown in Fig. 2. Chelex 100 (Bio-Rad, diameter $=70-150 \mu \mathrm{m}$ ), known as a chelate resin, is made of a polystyrene-divinylbenzene copolymer and is spherical as long as an observation is made under an optical microscope (Fig. 2a). Spherical silica gels (abbreviated as $s$-SG) with diameters of $20-40 \mu \mathrm{m}$ (Fig. 2b) were obtained from GL Science. We also used amorphous-shaped silica gels with sizes of $60-200 \mu \mathrm{m}$ (abbreviated as $a$-SG, Merck, Fig. 2c). These materials were used as supplied.

$\mathrm{Ru}(\mathrm{II})$-doped samples were prepared by soaking each (a)

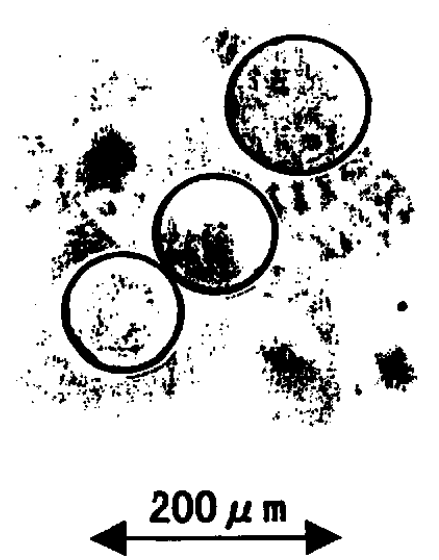

(b)

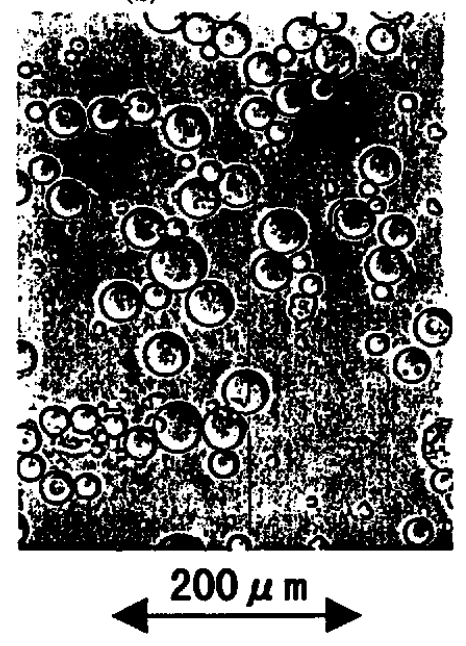

(c)

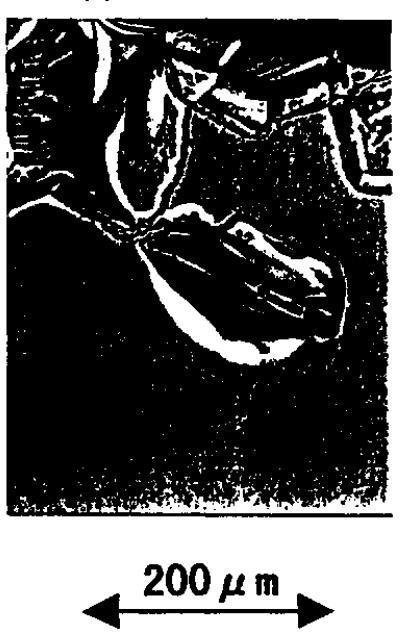

Fig. 2 Optical microphotographs of Chelex (a), $s$-SG (b), and $a$-SG (c) adsorbed with Ru(II). 
adsorbent $(1.6 \mathrm{~g})$ in an aqueous $\mathrm{Ru}(\mathrm{II})$ solution $(10 \mathrm{ml}$, $\left.1.4-7.0 \times 10^{-5} \mathrm{M}\left(\mathrm{mol} / \mathrm{dm}^{3}\right)\right)$ for $1 \mathrm{~h}$. For studying the effects of the dye-distribution characteristics on the DR spectrum, Chelex $(1.6 \mathrm{~g})$ was soaked in $100 \mathrm{ml}$ of an aqueous $\mathrm{Ru}(\mathrm{II})$ solution $\left(3.7 \times 10^{-5} \mathrm{M}\right)$ for $1 \mathrm{~h}-7 \mathrm{~d}$ (described later again). After soaking, the sample particles were collected by filtration, and then dried in air prior to experiments. Under the present experimental conditions, the total amount of $\mathrm{Ru}(\mathrm{II})$ is far below the adsorption/ion-exchange capacity of the adsorbent. Thus, all of the Ru(II) molecules dissolved in the mother solution were adsorbed by the adsorbents within $1 \mathrm{~h}$, as judged by the fact that the solution phase became transparent after soaking, which was confirmed spectroscopically. Using the densities of Chelex, $s$-SG, and $a$ SG to be $0.74,0.46$, and $0.57 \mathrm{~g} / \mathrm{cm}^{3}$, respectively, we calculated the amount of $\mathrm{Ru}$ (II) adsorbed on each material per unit volume ([Ru(II)]). The sample particles were filled in a quartz cuvette (path length, $10 \mathrm{~mm}$ ). For determining the light-scattering coefficient of the adsorbent, the thickness of a sample layer was varied from 5 to $0.1 \mathrm{~mm}$ by setting an appropriate number of black spacers in the cuvette. The thickness of the spacer layer was determined by a digital micrometer (Mitsutoyo Co., MDC-25M).

\section{Results and Discussion}

Effects of the size and shape of an adsorbent on DR spectra Figure 3a shows the DR spectra of $\mathrm{Ru}(\mathrm{II})$ adsorbed by Chelex, $s$-SG, and $a$-SG, together with the transmission spectrum of Ru(II) in water. Each DR spectrum exhibited a peak at around $450 \mathrm{~nm}$, characteristic of the metal-to-ligand charge-transfer band of a polypyridine ruthenium(II) complex, and the spectra were similar to the transmission spectrum of an aqueous solution of the complex $\left(\lambda_{\max }=452 \mathrm{~nm}\right.$, molar absorptivity $=1.4 \times 10^{4}$ $\left.\mathrm{M}^{-1} \mathrm{~cm}^{-1}\right){ }^{13}$ Although it is not clear from Fig. 3a, a close inspection of the spectra indicates that the band shape of the DR spectrum is slightly different from the transmission spectrum, particularly, in the wavelength

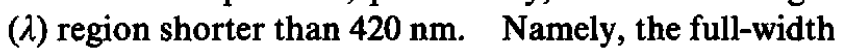
at half maximum (FWHM) of the DR spectrum was somewhat narrower than that of the transmission spectrum. In the case of DRS, since the $f\left(r_{\infty}\right)$ value is dependent on $S$ of a sample, the spectrum does not necessarily agree with the relevant transmission spectrum. A slight deviation of the DR spectrum from the transmission spectrum at $\lambda<420 \mathrm{~nm}$ will be thus ascribed to a change in $S$ between three samples, as discussed later again in detail.

The relationship between the amount of adsorbed $\mathrm{Ru}(\mathrm{II})([\mathrm{Ru}(\mathrm{II})])$ and $f\left(r_{\infty}\right)$ is shown in Fig. 3b. A good linear relationship between [Ru(II)] and $f\left(r_{\infty}\right)$ was obtained for each sample in the concentration range of $[R u(I I)]=0.4-2.0 \times 10^{-7} \mathrm{~mol} / \mathrm{cm}^{3}$. Since the absolute $f\left(r_{\infty}\right)$ value is below 3.5, the linear relationships in Fig. 3b are a reasonable consequence, as discussed above. The

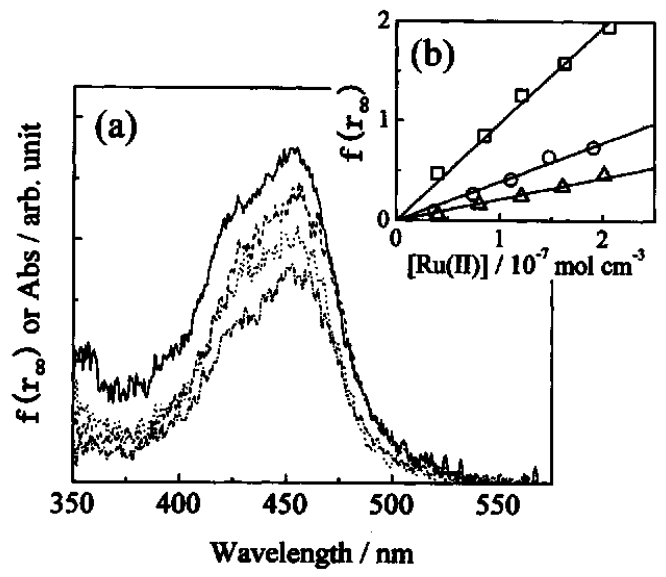

Fig. 3 (a) Diffuse reflectance and transmission spectra of $\mathrm{Ru}(\mathrm{II})$ on Chelex (broken line), $s$-SG (dotted line), a-SG (broken-dotted line), and in water (solid line). Both $f\left(r_{\infty}\right)$ and absorbance are in arbitrary units. (b) The concentration dependence of $f\left(r_{\infty}\right)$ determined at $450 \mathrm{~nm}$; Chelex $(\square) s-S G(\triangle)$, and $a$-SG $(O)$.

results in Fig. $3 \mathrm{~b}$ can be used for a quantitative analysis of $\mathrm{Ru}(\mathrm{II})$ on each adsorbent.

It is very important to note, however, that the slope of the plot does not agree with each other between three samples and, the sequence of the slope value was Chelex $>a$-SG $>s$-SG. The different $f\left(r_{\infty}\right)$ values between the samples, even at a common [Ru(II)], clearly indicate that the light scattering coefficient of the adsorbent, itself, is different. Generally, a decrease in the size of an adsorbent renders an increase in $S$ and, therefore, a decrease in $f\left(r_{\infty}\right)$. Phenomenologically, the decreasing order of the slope value in Fig. 3b almost agrees with that of the average size of the adsorbent: Chelex $(70-150 \mu \mathrm{m})>a-\mathrm{SG}(60-200 \mu \mathrm{m})>s-\mathrm{SG}(20-$ $40 \mu \mathrm{m})$. From the results, it is concluded that a direct comparison of $[\mathrm{Ru}(\mathrm{II})]$ or $f\left(r_{\infty}\right)$ between the different samples is not possible, although a quantitative analysis of [Ru(II)] on each adsorbed can be made on the basis of the linear relationship between $f\left(r_{\infty}\right)$ and [Ru(II)] in Fig. 3b. Thus, analyses of the data based on the $S$ value is necessary for a more detailed discussion.

Effects of the light-scattering coefficient of an adsorbent on the Kubelka-Munk function

The light-scattering coefficient of an adsorbent can be determined experimentally on the basis of Eq. (2) ${ }^{1,2}$,

$$
S \cdot d=R_{\mathrm{o}} /\left(1-R_{\mathrm{o}}\right)
$$

where $d$ represents the thickness of a sample layer. $R_{0}$ is defined as $R_{0}=R_{d} / R_{\infty}$, where $R_{d}$ or $R_{\infty}$ is the reflected light intensity from a sample with the thickness of $d$ or infinite $(\infty)$, respectively. Equation (2) indicates that a $d$ dependence of $R_{\mathrm{o}}$ affords the $S$ value of an adsorbent. $R_{\mathrm{o}}$ was determined for each sample by varying $d$ from 5 to $0.1 \mathrm{~mm}$. The results are plotted against $d$, as shown in 


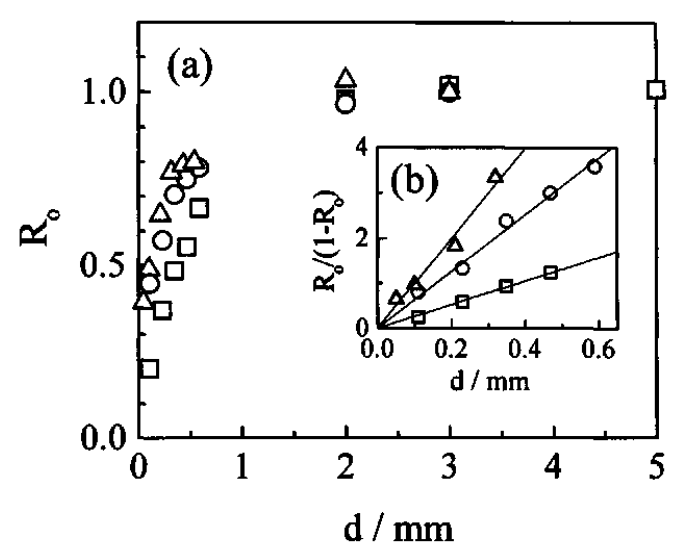

Fig. 4 Effects of the sample thickness on $R_{0}$ (a) and $R_{0} /$ $\left(1-R_{0}\right)(\mathrm{b})$. Chelex $(\square), s$-SG $(\Delta)$, and $a$-SG $(O) . \quad R_{0}$ was determined at $450 \mathrm{~nm}$.

Fig. 4a. $R_{0}$, determined at $\lambda=450 \mathrm{~nm}$, increased sharply along with an increase in $d$, and reached $c a .1 .0$ at $d=1$ $3 \mathrm{~mm}$. This indicates that the penetration depth for the incident light beam in the sample cuvette was 1-3 mm under the present experimental conditions. For the experiments in Fig. 3, we determined $f\left(r_{\infty}\right)$ for a sample thickness of $10 \mathrm{~mm}$. Therefore, the Kubelka-Munk approximation of $d=\infty$ determining $f\left(r_{\infty}\right)$ was satisfied in the present experiments.

Figure $4 \mathrm{~b}$ shows the relationship between $d$ and $R_{0} /$ $\left(1-R_{0}\right)$. As expected from Eq. (2), the plot falls on a straight line, and the $S$ value for each adsorbent could be determined as $26.3,62.9$, or $99.3 \mathrm{~cm}^{-1}$ for Chelex, $a$-SG, or $s$-SG, respectively (at $\lambda=450 \mathrm{~nm}$ ). Although the shape of Chelex or $s$-SG is spherical, the $S$ value of $s$-SG is much larger than that of Chelex, proving that smallersized particles $(s-\mathrm{SG} ; 20-40 \mu \mathrm{m})$ show a higher lightscattering coefficient. On the other hand, the size distribution of Chelex $(70-150 \mu \mathrm{m})$ and $a-\mathrm{SG}(60-200 \mu \mathrm{m})$ are very similar. However, the $S$ value was much larger for $a$-SG. It is easily concluded that the amorphous shape of $a$-SG (Fig. 2) is responsible for the high scattering coefficient.

In Fig. 5 , the $1 / S$ values, determined for each sample at several wavelengths, are plotted against the relevant $f\left(r_{\infty}\right)$ values (Eq. (1)). The linear relationship at a given wavelength indicates that the variation of the slope value in Fig. $3 \mathrm{~b}$ is essentially ascribed to that of $S$ between the samples, which is governed by the morphological shape and size of the adsorbent itself, as described above. Once the $S$ value of an adsorbent is determined, quantitative and direct analyses of any analytes can be made based on a relationship between $f\left(r_{\infty}\right)$ and analyte concentrations. Thus, the determination of the $d$ dependence of $R_{\mathrm{o}}$ is an essential basis for applications of DRS to various dye-doped materials. For dye-doped systems, the present approach is more convenient compared to the conventional method using $\mathrm{MgO}$.

It is worth discussing the wavelength dependence of the

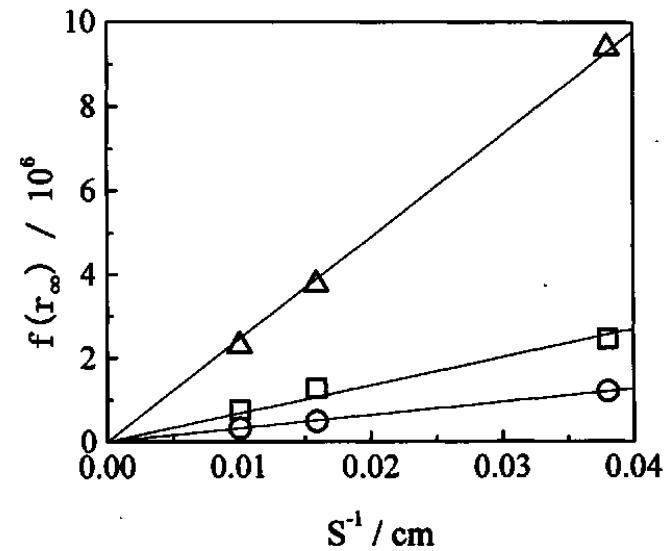

Fig. 5 Relationships between $f\left(r_{\infty}\right)$ and $S^{-1}$ determined at 400 $(\square), 450(\triangle)$, and $500 \mathrm{~nm}(\mathrm{O})$.

slope value in Fig. 5. According to Eq. (1), the slope value corresponds to $K$ of $\mathrm{Ru}(\mathrm{II})$. Thus, $K$ was determined to be $9.1 \times 10^{7}, 2.6 \times 10^{8}$, and $3.3 \times 10^{7} \mathrm{~cm}^{-1}$ at 400,450 , and $500 \mathrm{~nm}$, respectively. As noted in the preceding section, $K$ should be proportional to the relevant absorptivity determined by transmission spectroscopy. In order to test whether this is correct or not in the present system, the following estimations were performed. The transmission spectrum of $\mathrm{Ru}(\mathrm{II})$ in water (Fig. 3a) affords the ratio of absorbance $(A)$ at $450 \mathrm{~nm}$ to that at 400 or $500 \mathrm{~nm}$ to be $A(450 / 400)=2.56$ or $A(450 / 500)=7.87$, respectively. Similarly, the ratio of $K$ at $450 \mathrm{~nm}$ to that at 400 or $500 \mathrm{~nm}$ can be calculated from the data in Fig. 5 as $K(450 / 400)=2.86$ or $K(450 /$ $500)=7.88$, respectively. Since $A(450 / 500)$ agrees very well with $K(450 / 500)$, it is concluded that the absorbance ratio of $\mathrm{Ru}(\mathrm{II})$ is correctly reflected on the $\mathrm{DR}$ spectrum. On the other hand, $K(450 / 400)$ was larger than $A(450 /$ 400 ), indicating that the $K$ value at $400 \mathrm{~nm}$ was not determined by the absorptivity of $\mathrm{Ru}(\mathrm{II})$ alone. This demonstrates that the $\lambda$ dependence of $S$ should be considered in order to explain the present results.

The wavelength dependence of $S$ for each Ru(II)/ adsorbent system is shown in Fig. 6 . The $S$ values for Chelex and $a$-SG were almost independent of $\lambda$ at 420 $550 \mathrm{~nm}$, while those increased slightly at $\lambda<420 \mathrm{~nm}$. For $s-\mathrm{SG}$, on the other hand, although the data are somewhat scattered, the $S$ value seems to gradually increase at $\lambda<450 \mathrm{~nm}$. Thus, the $\lambda$ dependence of $S$ more or less influences the band shape and FWHM of the DR spectrum.

A close inspection of the spectra in Fig. 3a indicates that the FWHM of Ru(II) on the adsorbent (Chelex, $s$ $\mathrm{SG}$, or $a-\mathrm{SG})$ is slightly narrower $\left(c a .3200 \mathrm{~cm}^{-1}\right)$ than that of the transmission spectrum in water (ca.3600 $\mathrm{cm}^{-1}$ ). The adsorption of $\mathrm{Ru}(\mathrm{II})$ on polymer beads or silica gels may alter the electronic state of $\mathrm{Ru}$ (II), leading to the slight change in FWHM. According to the data in Fig. 6, however, an increase in the $S$ value of 


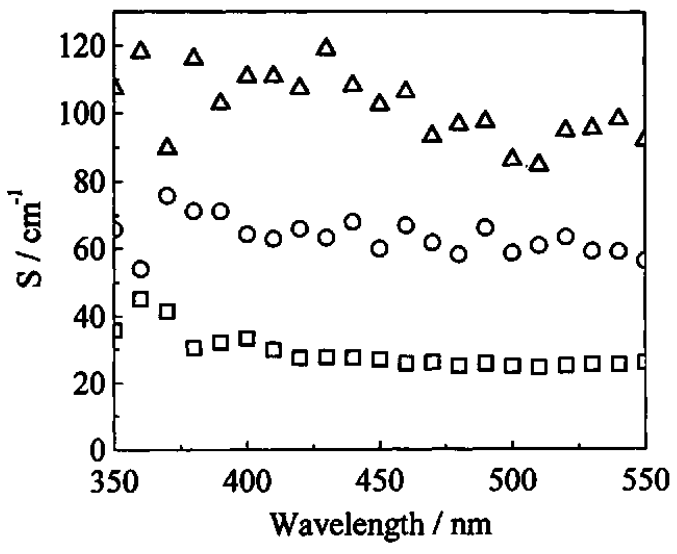

Fig. 6 Wavelength dependencies of the light-scattering coefficients of Chelex ( $\square), s-S G(\Delta)$, and $a-\operatorname{SG}(O)$.

the adsorbent at $\lambda<420 \mathrm{~nm}$ (Chelex and $a$-SG) or $\lambda<450 \mathrm{~nm}(s-\mathrm{SG})$ leads to a relatively small $f\left(r_{\infty}\right)$ value, compared to that at $\lambda>420$ or $450 \mathrm{~nm}$. This is also a probable reason for the band narrowing of the DR spectrum. The DR spectral band shape is dependent on the shape and morphology of the adsorbent itself.

\section{Effects of dye-distribution characteristics in an adsorbent on} DR spectra

Besides the size and shape of an adsorbent on the DR spectrum, the distribution characteristics of a dye inside an adsorbent might be an another factor governing $f\left(r_{\infty}\right)$. However, such a study has never been explored, since there has been no means to investigate three-dimensional distribution characteristics of a solute in a single solid particle. Previously, we reported on in situ measurements of ion-exchange processes of a dye ( $R u(I I)$ or Rhodamine B) in single polymer microparticles on the basis of confocal fluorescence microspectroscopy and laser trapping-absorption microspectroscopy, and demonstrated that the spatial distribution of the dye in the particle varied with the soaking time of the particles in a dye solution. ${ }^{14,15}$ In order to study the effects of the dye-distribution characteristics on the DR spectra, we prepared several $\mathrm{Ru}(\mathrm{II}) /$ Chelex samples with different dye distributions in a single Chelex particle by changing the soaking time $(1 \mathrm{~h}-7 \mathrm{~d})$, and measured the relevant DR spectra.

Figure 7 shows the relationship between $f\left(r_{\infty}\right)$ at $450 \mathrm{~nm}$ and the soaking time $(t)$ of Chelex in an aqueous $\mathrm{Ru}(\mathrm{II})$ solution $\left(3.7 \times 10^{-5} \mathrm{M}\right)$. At $t<1 \mathrm{~d}, \mathrm{Ru}(\mathrm{II})$ is expected to be adsorbed by the surface layer of the particle and the dye adsorption proceeds into the inner volume upon prolonged soaking up to 1 week. ${ }^{15}$ Thus, the dye distribution chatacteristics in the particle is expected to be different with $t$. As can be seen in Fig. 7, however, $f\left(r_{\infty}\right)$ was essentially independent of the soaking time, and the DR signal was not governed by the dyedistribution characteristics in the particle. This implies that the incident light beam propagates into all single-

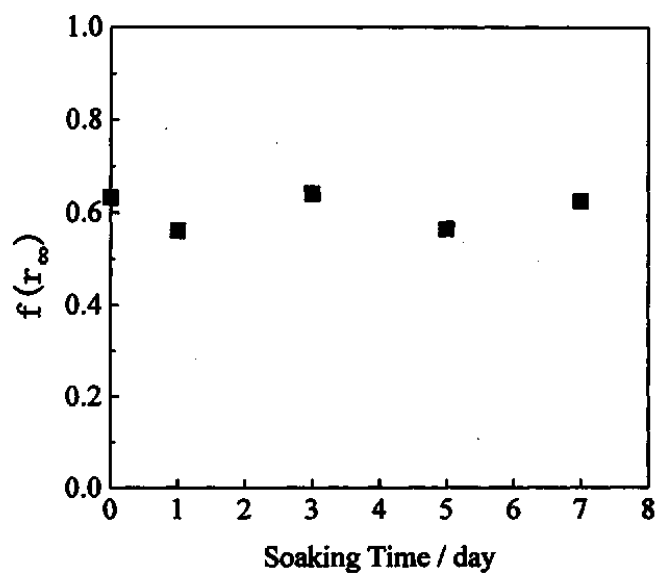

Fig. 7 Effects of the dye-distribution characteristics in Chelex on determined at $450 \mathrm{~nm}$. See also the main text.

particles, and DRS does not provide any information on the dye distribution characteristics in each particle. According to the present discussion, however, the penetration depth of the incident light beam and, thus, the sensing layer by DRS, is dependent on the shape and size of the adsorbent as well as on the probing wavelength, since the $S$ value is dependent on these factors. Namely, the sensing layer becomes thinner in a particle or in a wavelength region possessing a larger light-scattering coefficient.

DRS is a quite unique and indispensable method for analyzing various solid materials. As discussed in the present paper, however, since the light-scattering coefficient is different between materials depending on their size, shape, and morphology, care should be taken to compare the data obtained for different samples. Also, it has been shown that DRS cannot provide any information on the spatial distribution of a dye in materials. For materials possessing inhomogeneous dye distributions, we think that a complementary study by DRS, laser trapping-microspectroscopy, and confocal fluorescence microspectroscopy is quite important, since the microspectroscopy methods provide information about a spatial distribution of a solute in a single microparticle. Such systematic studies will engage an understanding of the limitations and/or further applicabilities of DRS.

This study was partly supported by a Grant-in-Aid from the Ministry of Education, Science, Sports and Culture (08404051).

\section{References}

1. G. Kortlum, "Reflectance Spectroscopy; Principles, Methods, Applications", Springer-Verlag, Berlin, 1969.

2. W. WM. Wendland and H. G. Hecht, "Reflectance Spectroscopy", Interscience Publishers, New York, 1966.

3. A. Mandelis (ed.), "Principles and Perspectives of 
Photothermal and Photoacoustic Phenomena", Elsevier, New York, 1992.

4. P. R. Brown and B. T. Beauchemin, J. Liq. Chromatogr., 11, 1001 (1988).

5. M. Blanco, J. Coeello, H. Iturriaga, S. Maspoch and C. de la Pezuela, Anal. Chim. Acta, 333, 147 (1996).

6. F. Boroumand, H. van den Bergh and J. E. Moser, Anal. Chem., 66, 2260 (1994).

7. S. Langer, R. S. Pemberton and B. J. Finlarson-Pitts, J. Phys. Chem. A, 101, 1277 (1997).

8. T. Asahi, Y. Matsuo and H. Masuhara, Chem. Phys. Lett., 256, 525 (1996).

9. D. P. Colombo Jr. and R. M. Bowman, J. Phys. Chem., 100, 18445 (1996).

10. X. Liu, G. Zhang and J. K. Thomas, J. Phys. Chem., 99, 10024 (1995).
11. F. Wilkinson and G. P. Kelly, in "Photochemistry on Solid Surfaces", ed. A. Ampo and T. Matsuura, p. 30, Elsevier, Amsterdam, 1989.

12. J. Klaas, G. Schulz-Ekloff and N. I. Jaeger, J. Phys. Chem. $B, 101,1305$ (1997)

13. K. Kalyanasundaram, Coord. Chem. Rev., 46, 159 (1982).

14. N. Kitamura, M. Hayashi, H.-B. Kim and K. Nakatani, Anal. Sci., 12, 49 (1996).

15. H.-B. Kim, M. Hayashi, K. Nakatani, N. Kitamura, K. Sasaki, J. Hotta and H. Masuhara, Anal. Chem., 68, 409 (1996).

(Received May 2, 1997) (Accepted June 19, 1997) 